\title{
Urethral Catheterization and Chronic Irritation; Are Our Patients at Risk of Malignancy
}

\author{
Haitham Abdelmoteleb ${ }^{1 *}$, Amr Hawary ${ }^{1}$
}

Great Western Hospital, Swindon, UK

Received: July 1, 2017; Accepted: August 14, 2017; Published: August 23, 2017

*Corresponding author: Haitham Abdelmoteleb, Great Western Hospital, Swindon, UK. E-mail: haitham.abdelmoteleb@yahoo.com

\begin{abstract}
The introduction of indwelling catheters has been very beneficial to patients with chronic debilitating diseases. However, the long-term use of catheters isn't problem free. Guidelines regarding follow-up of patients with chronic indwelling catheters are not clear and need justifying.
\end{abstract}

Keywords: Indwelling Catheter; Bladder Tumour

\section{Abbreviations:}

Ciucs: Chronic Indwelling Catheters; SCI: Spinal Cord Injury; SCC: Squamous Cell Carcinoma; TCC: Transitional Cell Carcinoma; ISC: Intermittent Self-Catheterization; UTI: Urinary Tract Infections.

\section{Introduction}

The increasing use of Chronic Indwelling Catheters (CIUCs) isn't free of problems. Although it is a very valuable tool and sorts lots of issues, the complications that arise due to its prolonged use are debilitating to the patients.

CIUCs are known as a risk factor for bladder cancer especially when used in Spinal Cord Injury (SCI) patients. None the less, there has been an established association to bladder cancer in non-SCI patients having CIUCs compared to the general population [1]. Squamous cell carcinoma (SCC) of the bladder usually occur secondary to bilharzia in the Middle East, Southeast Asia, and South America, where schistosomiasis is endemic and accounts for $59 \%$ to $75 \%$ of bladder SCC cases. However, in western regions, SCC of the bladder usually constitutes only $1.2 \%$ to $4.5 \%$ of all bladder cancers, and these are usually secondary to prolonged indwelling catheters, especially in SCI patients.

SCC of the bladder is the most common type of bladder cancer presenting in patients with CIUCs. However, other studies have reported Transitional Cell Carcinoma (TCC) to be the predominant type in their series and adenocarcinoma of the bladder to be a less common presentation [2]. Bearing in mind that the mechanism of development of bladder cancer in CIUCs patients is chronic irritation of the bladder and recurrent Urinary Tract Infections (UTI), it is not surprising to find reports of SCC in patients performing Intermittent Self-Catheterization (ISC) [3].

The problem in SCI patients is that the majority of those patients have impairment in the sensory system thus, they are asymptomatic. Non-SCI patients, on the other hand, retain their sensory input thus could present early. Red flag signs for a low threshold for a cystoscopy are haematuria, recurrent Urinary Tract Infections (UTI), recurrent catheter blockage, bladder stones, and debris [4].

Despite acknowledging cost effectiveness, NICE does not recommend cystoscopic surveillance in individuals with neurogenic lower urinary tract dysfunction in general [5]. Individual institutionalized protocol for surveillance of SCI does include annual cystoscopy in SCI patients with CIUCs 10 years after insertion then every 1-2 years depending on the findings from previous cystoscopy and patients' presentation.

\section{Conclusion}

In the absence of guidelines for the follow-up of non-SCI patients having CIUCs, a high index of suspicion should be kept in mind regarding the follow-up of these patients. Although rare, bladder cancer is a potential long-term complication in patients with CIUCs.

\section{References}

1. Ho CH, Sung KC, Lim SW, Liao CH, Liang FW, Wang JJ, et al. Chronic Indwelling Urinary Catheter Increase the Risk of Bladder Cancer, Even in Patients Without Spinal Cord Injury. Medicine (Baltimore). 2015;94(43):e1736. doi: 10.1097/MD.0000000000001736

2. West DA, Cummings JM, Longo WE, Virgo KS, Johnson FE, Parra RO. Role of chronic catheterization in the development of bladder cancer in patients with spinal cord injury. Urology. 1999;53(2):292-297.

3. Casey RG, Cullen IM, Crotty T, Quinlan DM. Intermittent selfcatheterization and the risk of squamous cell cancer of the bladder: An emerging clinical entity? Can Urol Assoc J. 2009;3(5):E51-54.

4. El Masri y WS, Patil S, Prasanna KV, Chowdhury JR. To cystoscope or not to cystoscope patients with traumatic spinal cord injuries managed with indwelling urethral or suprapubic catheters? That is the question! Spinal Cord. 2014;52(1):49-53. doi: 10.1038/sc.2013.119

5. Urinary incontinence in neurological disease: assessment and management | Guidance and guidelines | NICE. 2017 